\title{
A Functional Modeling Framework for Representing and Comparing Models of Consciousness or Cognition
}

\section{Andy E. Williams, awilliams@nobeahfoundation.org}

\begin{abstract}
Borrowing techniques from complex systems and software engineering, this paper defines a Functional Modeling Framework to provide a simple common mechanism for representing models of consciousness or cognition that is independent of assumptions made by any specific model. All models of consciousness or cognition should of course fit into the complete set of functions of what consciousness, or cognition can do. Through attempting to represent all such functions (both computable and non-computable), this framework attempts to gain the capacity to represent all models of consciousness or cognition, even where the implementation mechanisms of those functions are unknown. Enabling different models of consciousness or cognition to be more easily compared is intended to enable research on consciousness and cognition to more reliably converge on a single understanding, even across massively collaborative research projects spanning multiple disciplines. Furthermore, if biological functionality can be considered as dynamically stable where it is persistent, then in aiming to provide a common approach for representing and comparing the functionalities of consciousness or cognition as dynamically stable systems, this framework provides a set of features through which it may be possible to see commonality between the functional components of a wide range of other dynamically stable biological or non-biological systems. If such commonality does exist, and if, as proposed, that commonality reflects deeper underlying physical and mathematical principles, then representing any researcher's model of consciousness or cognition within this framework might help reveal applications of that researcher's model across a wide range of other domains of study.
\end{abstract}

\section{Keywords}

functional modeling; functionalism; human-centric; model of consciousness; model of cognition

\section{Introduction}

The number of models of consciousness or cognition in existence today may already be unmanageably large in comparison to the ability of any one individual to understand and choose between all of them. Rather than attempting to actually do such a comparison and make such a choice, a more scalable approach might be to find a way to automate such comparisons through a universal mechanism for defining the problem of consciousness or cognition, and through a universal mechanism for measuring fitness in solving that problem, so that it is more possible to compare the fitness of all available models to discover which component of each model offers the best understanding. Functional modeling approaches have long been used in various engineering domains [21], [22], [23],[24],[25],[26],[27], [28], because functional components with well defined interfaces remove the need to understand other work, creating the capacity for teams spread across a great variety of different disciplines to productively work on a single complex problem. The human-centric Functional Modeling Framework (FMF) described in this paper aims to provide a methodology for defining all conceptual entities according to the functions of those entities that can be observed in conscious human awareness. This includes the defining the functions of the human organism as required to enable consciousness or cognition to be clearly differentiated from other functions. Defining simple functional models of complex problems like consciousness or cognition, is intended to enable any models of consciousness or cognition proposed as solutions to be represented and compared in these common terms. Where in the past the task of understanding the many models of consciousness or cognition across the great variety of different disciplines in which they have been defined, has been too difficult to coherently 
converge on a single self-consistent model, a functional modeling approach is intended to create the potential capacity to accelerate research through enabling such convergence.

Of course even the best comparison mechanism is useless, if such comparisons simply aren't made, so part of understanding which components of which models offer the best understanding is finding a way to ensure such a comparison is actually performed. Rather than defining a system of enforcement based on rules that can be broken, a more robust approach might be to define a system in which choice of solution, choice of method of enforcement, or choice of anything else, naturally converges on whatever works best. For this purpose the FMF proposes a process for adapting through cooperation.

Cooperation in the FMF is one of several adaptive domains. This adaptive domain provides principles of intelligent cooperation (also representable as processes of cooperation) to help ensure all cooperation converges on optimal use of any resource available to the group. This potential to adapt to become more fit means that the framework itself is fluid and not entangled with any set definition for any of these elements. In other words, this cooperation means that all parts of the framework are intended to be replaceable by any other implementation that demonstrates greater fitness in representing observed human functionality.

The FMF proposes that the human system can be represented in terms of a set of adaptive processes that forms a hierarchy in terms of order of evolution. As mentioned, one of these domains of adaptation is adaptation through cooperation. Other proposed domains of adaptation are "action" (the ability to percieve the organism's current state, and to navigate to a different state). There is also the domain of adaptation through "reasoning", which is defined in the FMF as being implemented by the cognitive system (the mind).

Each domain is defined as being implemented by a functional system, or by a functional component that might be present in multiple functional systems. Each functional component or functional system is represented as a set of functions. Some of these functions are computable in that they have a predetermined output for a given input. Other functions are not computable in that a given input does not produce a predetermined output, though outputs are still proposed to be confined to the same "space" as the input so that the system may continue to function in a stable way.

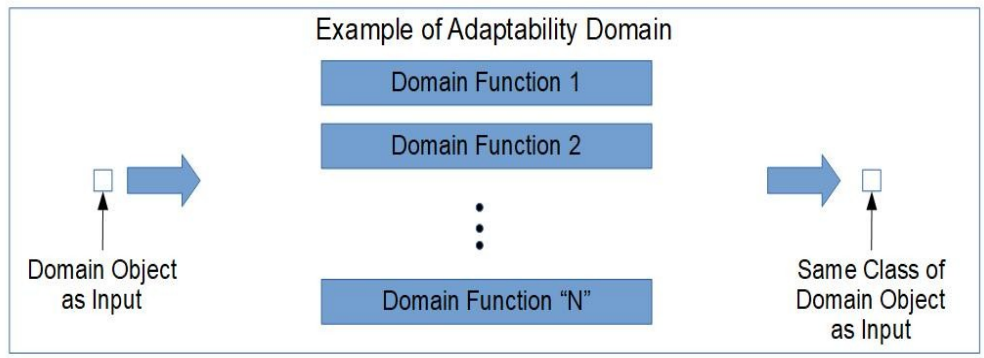

Figure 1: Each adaptability domain consists of a set of functions. The FMF proposes that all processes in each adaptability domain can be composed from a set of basic functions, each of which operates on a single category of entity within the domain (a domain object).

In any case, all the states accessible to the system through those functions are then defined as forming a "functional state space". The dynamics of the system through that state space are then described in terms of the dynamics between those functions. 


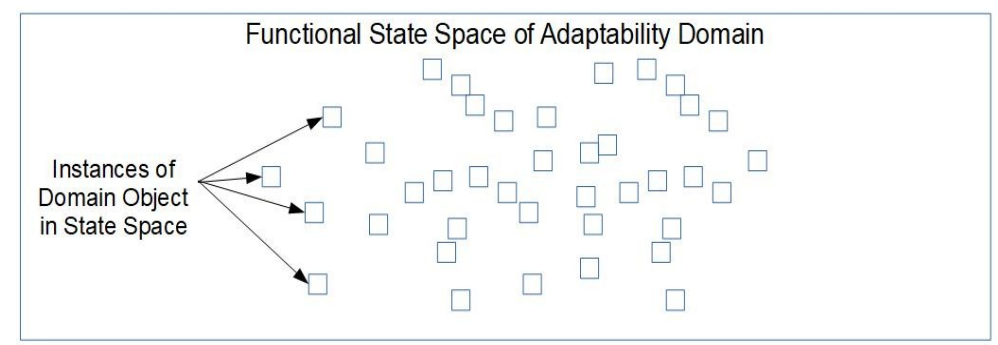

Figure 2: Figure 3: All the states accessible through the functions form a functional state space for the adaptive domain.

A model defined this way might then provide great insight into the workings of consciousness, cognition, or other systems from an understanding of those dynamics, without even understanding how the functions themselves are implemented. In the consciousness or cognitive system this functional state space aims to describe all the states the system can experience. In this way, the FMF aims to provide a set of functions in terms of which models of consciousness or cognition may be represented, and to provide a set of adaptive processes by which functional models might adapt to become more fit. These adaptive processes are as listed below:

\begin{tabular}{|l|l|}
\hline Adaptive Processes (Basic Life Processes) \\
\hline Life Process & Name \\
\hline L1 & Homeostasis \\
\hline L2 & Autopoiesis (Reproduction) \\
\hline L3 & Growth (Maximize Outcomes) \\
\hline L4 & Evolve \\
\hline L5 & Act \\
\hline L6 & Cooperation (Between Functional Units) \\
\hline L7 & Motivation \\
\hline L8 & Reason \\
\hline
\end{tabular}

Table 1: Adaptive processes.

Each adaptive domain in the hierarchy can potentially interact. For example, defining the functions of the homeostasis domain to include breathing, then all reproductive processes (one domain above in the hierarchy) occur within the process of executing these homeostasis processes. Similarly, reproduction occurs within growth, and so forth up the hierarchy. Action in this framework is defined as occurring when there are multiple paths to achieve stability in fitness space and the system must "choose" a path as it's "action", as opposed to having a single path and simply "responding" as in plants. In the lower domains (L1 to L4, L5 at the cellular level, and L6) the functional state space corresponding to the fitness space of the domain is a space in which each point represents a different set of physical properties or other environmental signals. In the upper domains (L5 at the physical system level, L7, L8, and L5 at the consciousness system level) the functional state space is a perceptual space in which each point represents a perception in that domain. Action in these domains is then the capacity to detect 
position in a perceptual space of a domain and to choose a path through perceptual space from that position. From this perspective reflexive action might occur at the level of single cells, but selfconscious action requires movement through a perceptual space, and occurs only in each functional system (body, emotions, mind, and consciousness).

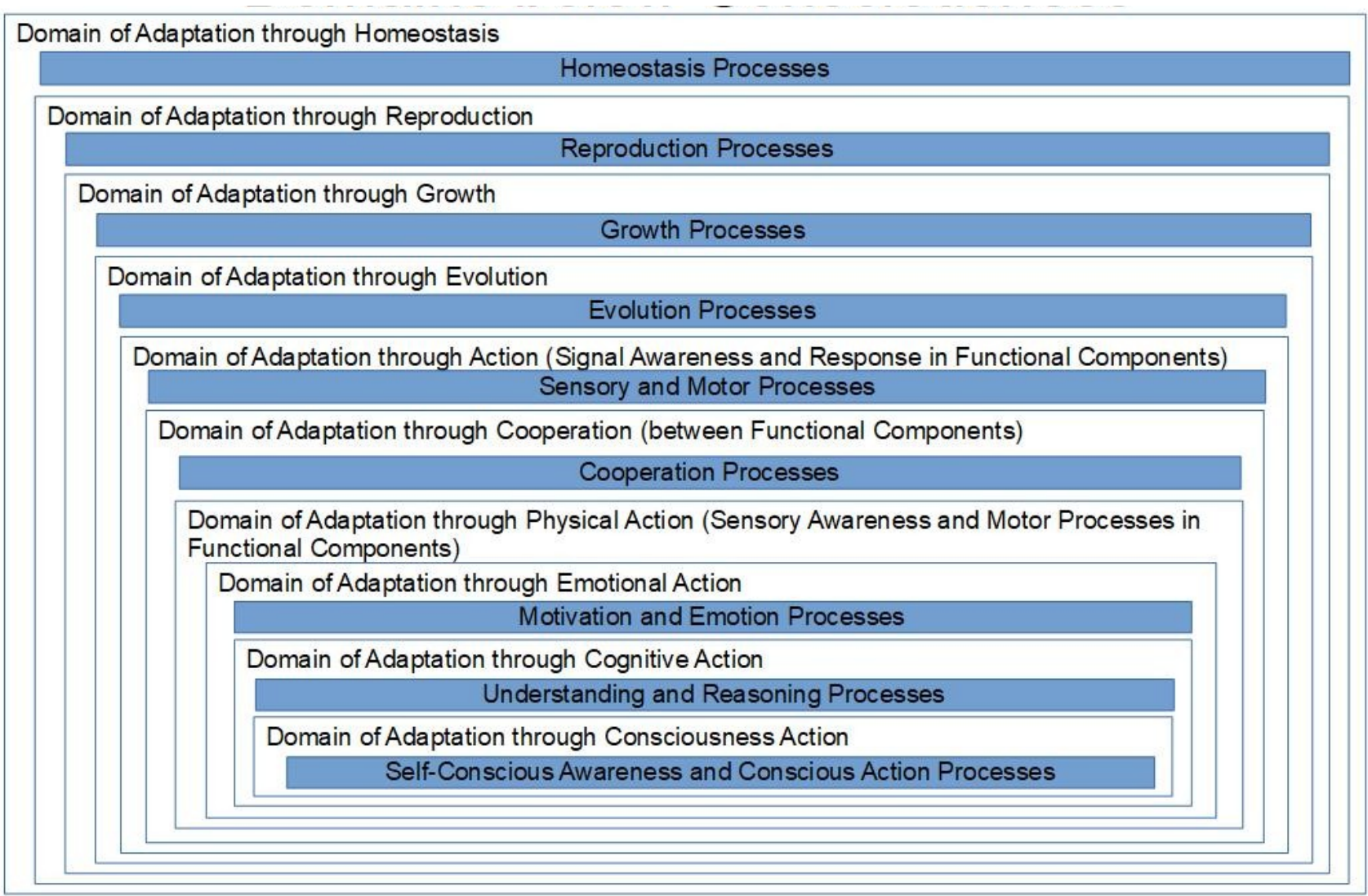

Figure 3: Interaction between adaptability domains.

In the cognitive domain, as described later in this paper, the FMF represents anything the cognitive system can do (i.e. all reasoning or understanding processes) as compositions of seven cognitive functions. The lower cognitive functions (F1 to F3) process neural signals into concepts. The higher cognitive functions (F4 to F7) process concepts as inputs and produce concepts as outputs. In this way the cognitive system navigates a functional state space (the "conceptual space") in which reasoning or understanding processes trace paths between concepts. In attempting to define all the states the cognitive system can experience, and all the functions the cognitive system can do, the FMF aims to gain the ability to represent any model of cognition. Similarly, in the FMF all processes in the consciousness are represented as compositions of seven awareness functions (F1 to F7). Both the possible inputs to, and outputs from, those consciousness functions belong to the same category of entity (an awareness), and are therefore confined to that awareness state space, so that through the functions themselves, as well as through the dynamics between the functions, this functional modeling approach can potentially describe all that the consciousness can do.

The FMF also defines the attributes it proposes that a model of consciousness must have in order to have the capacity to represent self-awareness, and defines attributes it proposes a model of cognition must have in order to have the capacity to represent general problem solving ability or true intelligence. In practice, the FMF has been used to compare some leading models of consciousness [8], as well as having been used to define a mathematical model of consciousness that according to these attributes 
has the potential capacity for self-awareness, and a mathematical model of cognition with the potential capacity for general problem solving ability[1],[2]. These models in turn incorporate mathematical patterns of stability that have been suggested to characterize all life in that these patterns are proposed to exist in all stable functions of biological organisms. This stability has in turn been suggested to reflect fundamental physical laws [59].

In summary, defining a functional state space in this functional modeling approach enables complex systems to be described in terms of their observed functions, and in terms of the observed constraints on the dynamics between the functions, even where the implementations of the functions themselves are unknown. Human-centric functional modeling specifically facilitates the modeling of human systems, such as consciousness or cognition, in which lack of understanding of how such functions are implemented is certainly the case. Having defined these consciousness and cognitive functions, adaptive processes, and processes of cooperation in the FMF, then whether or not any given model of consciousness or cognition implements any one of these elements, the FMF can still be used to compare that model of consciousness with other models of consciousness and can still be used to compare that model of cognition with other models of cognition, not by comparing implementations of this functionality that might not yet exist for some models, but simply on the basis of whether or not that functionality is incorporated in the model being compared.

\section{Organization of this Paper}

This paper attempts to provide an overview explanation of all the parts of this framework and how they fit together. These concepts are expanded in further detail in a set of books currently being drafted. This framework is intended to provide a single approach that might be used by a wide range of disciplines to understand models of consciousness or cognition. Creating a single paper that provides not only an explanation of the framework, but that also provides sufficient detail to satisfy the needs of all potential disciplines that might leverage the framework is likely too ambitious to be reasonable. But in order to be successful at the goal of providing an overview of how the framework models the functions of consciousness and cognition, how those functions are integrated in the human system, and how all the proposed functional components share common features that hint at a single evolutionary path, it is useful to provide an overview of how the paper aims to achieve the goal of tying all these elements together to enable the reader to clearly recognize these relationships amidst all the information.

This paper begins by summarizing the representation of the functions of cognition in the FMF, and then attempts to justify why elements of that representation have been reused in representing the functions of consciousness in the FMF. The representation in the FMF of the other functional systems (body, and emotions), including the five senses of the body, are then summarized, along with the integration of those functional systems in a single self-consistent functional model representing the whole of the human system. The paper then comments on why there are eight types of awareness in this framework. To elaborate, the paper then explores how the framework has been used to define functional models of properties that humans intuitively understand, but for which the mechanisms implementing those functions have been difficult to define, such as intelligence, and conscious self-awareness.

Having explored the FMF, the paper then summarizes the properties that models of consciousness or cognition are proposed to be required to have in order to be considered valid and complete from the perspective of the FMF.

\section{Representing Models of Cognition}

The FMF proposes that seven functions can be used to represent the complete set of cognitive functions that occur on the input processing path of the cognitive system (processing to accomplish cognition of 
sensory or other input). This set of input cognitive functions is proposed to be assembled into sequences the cognitive system might use to receive understanding in terms of concepts (understanding meaning the process that enables comprehension of the sentence "the quick brown fox jumped over the lazy dog"). On the output path, the set of output cognitive functions is proposed to be assembled into sequences the cognitive system might use to direct understanding through reasoning (reasoning meaning the process that enables answering the question "what fox jumped over what dog?").

\begin{tabular}{lll} 
Functional Unit & \multicolumn{1}{c}{ Functional Units in $\begin{array}{c}\text { Systems of Cognition } \\
\text { Output Function }\end{array}$} \\
F1 to F3 & $\begin{array}{l}\text { Create Concept } \\
\text { STORE (Store Concept) }\end{array}$ & $\begin{array}{l}\text { Create Signals from Concept } \\
\text { DECOMPOSE STORAGE (Determine Concept } \\
\text { in Storage Function) }\end{array}$ \\
F4 & RECALL (Recall Concept) & $\begin{array}{l}\text { DECOMPOSE RECALL (Determine Concept in } \\
\text { Recall Function) }\end{array}$ \\
F5 & $\begin{array}{l}\text { DECOMPOSE PATTERN (Detect Concept in } \\
\text { PETECT PATTERN (Detect }\end{array}$ & $\begin{array}{l}\text { Pattern) } \\
\text { Pattern e.g. AND, OR, NOT, }\end{array}$
\end{tabular}
between Concepts.)

DETECT SEQUENCE (Detect

Sequence of Patterns between Concept)

DECOMPOSE SEQUENCE (Detect Concept in Sequence of Patterns)

FS

COGNITIVE AWARENESS (Choose Problem to Solve and Understanding or Reasoning Process to Solve it)

Table 2: Computable cognitive functions F1 to F7, and non-computable cognitive function FS.

Functional unit F7 is defined as detecting a sequence of patterns. Human reasoning has been characterized as consisting of intuitive reasoning and rational methodical reasoning [60]. Assuming that intuition can be modeled as estimating a solution from past patterns, then any intuitive reasoning can be represented in a functional model as a form of pattern detection.. Functional unit F6 is defined as detecting a pattern such as a logical relationships between concepts. Assuming that rational methodical reasoning can be modeled as a sequence of reasoning operations, that in turn can be expressed in terms of any complete set of logical relationships between concepts, then all rational methodical reasoning can potentially be represented with these functions. Since the set of functions AND, OR, as well as NOT can represent all logic and is therefore a Turing complete set of operations, this paper proposes that any logic, and therefore the logic in any rational methodical reasoning process, can be represented in a functional model in terms of a function to detect patterns (detectable by F6) where those patterns in turn might represent any logic in a Turing complete set of logic functions (whether or not that set consists of the functions AND, OR, and NOT), and in terms of a pattern in the sequence of those patterns (detectable by F7).

From F4 to F7, the order of the hierarchy represents the order the functional components are proposed to have evolved in. Whether this order might also reflect tendencies for certain functional units to occupy physical positions relative to other functional units in the brain has not been thoroughly examined. For example, functional units F1 might tend to be on the outer surface, and functional units F7 might tend to be closer to the core. However in the FMF this hierarchical order does not determine the order of functional units in the processing path of any reasoning. Multiple instances of each of these functional units, are together proposed to have the capacity to represent any intuitive or rational methodical reasoning process. A conceptual physical model of the brain indicating the connections between such functional units has not yet been fully elaborated. Such a model might connect these 
functional units into paths not shown in the pie slice of a conceptual physical model below, through some unknown mechanisms not specified here.

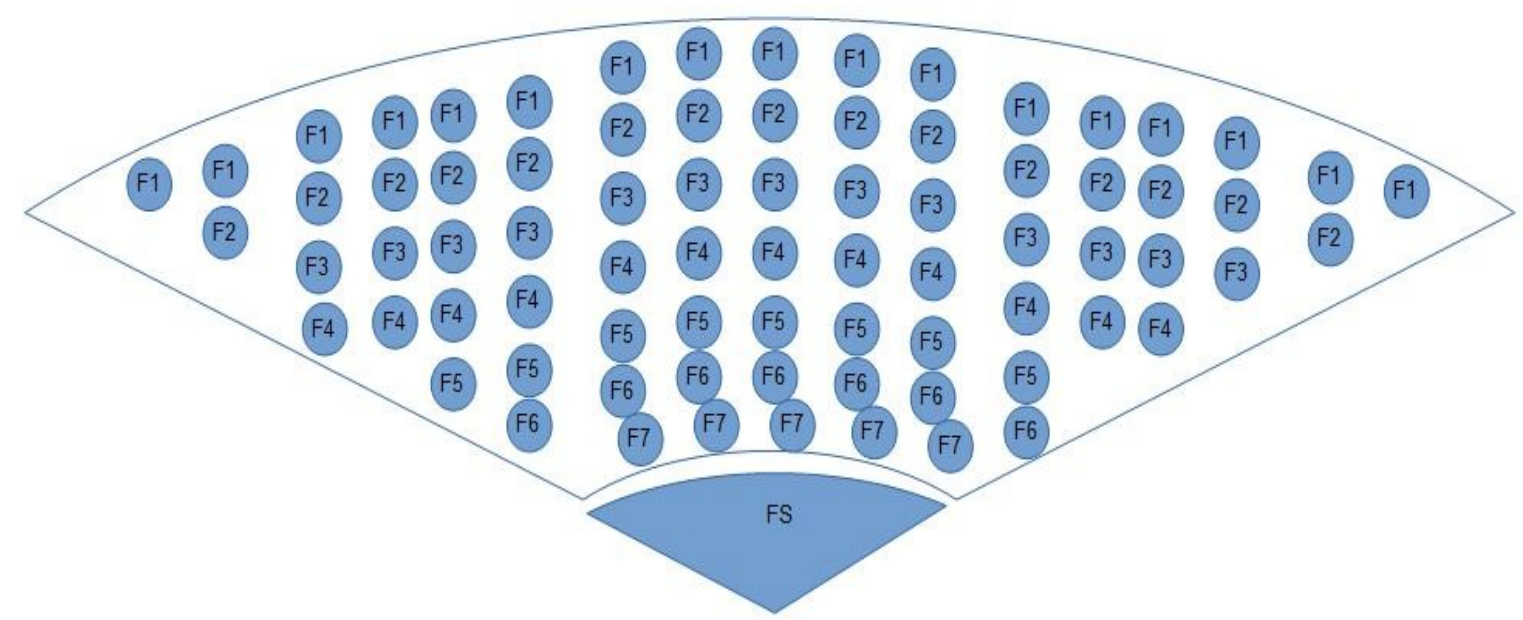

Figure 4: Pie slice through conceptual physical model of the system of cognition.

As an example, consider how the chain of understanding in the sentence "The quick brown fox jumped over the lazy dog" might be represented with this set of cognitive functions, as well as with the conceptual space and other elements defined by the framework. Firstly, in the FMF all reasoning or undertanding are represented as paths between concepts in conceptual space. Concepts are defined by their position in conceptual space. And the position of any concept in conceptual space is defined by its relationships with other concepts. The relationships that exist between one concept and another depend on the mind's perspective. And that perspective in turn reflects relative position in conceptual space. In the diagram below of a region in conceptual space, the direction of the large hollow arrows with the dashed blue lines represent the line of reasoning defining a relationship between two concepts, where that relationship is in turn defined by a given perspective. There are a multitude of relationships connecting the fox to entities (concepts) that define its various properties. For example, from the perspective of the large hollow dashed blue arrow defining the line of reasoning representing the perspective of a "mouse" the fox is "large". From the perspective of the large hollow dashed blue arrow defining the line of reasoning representing a "horse" the fox is "small". 


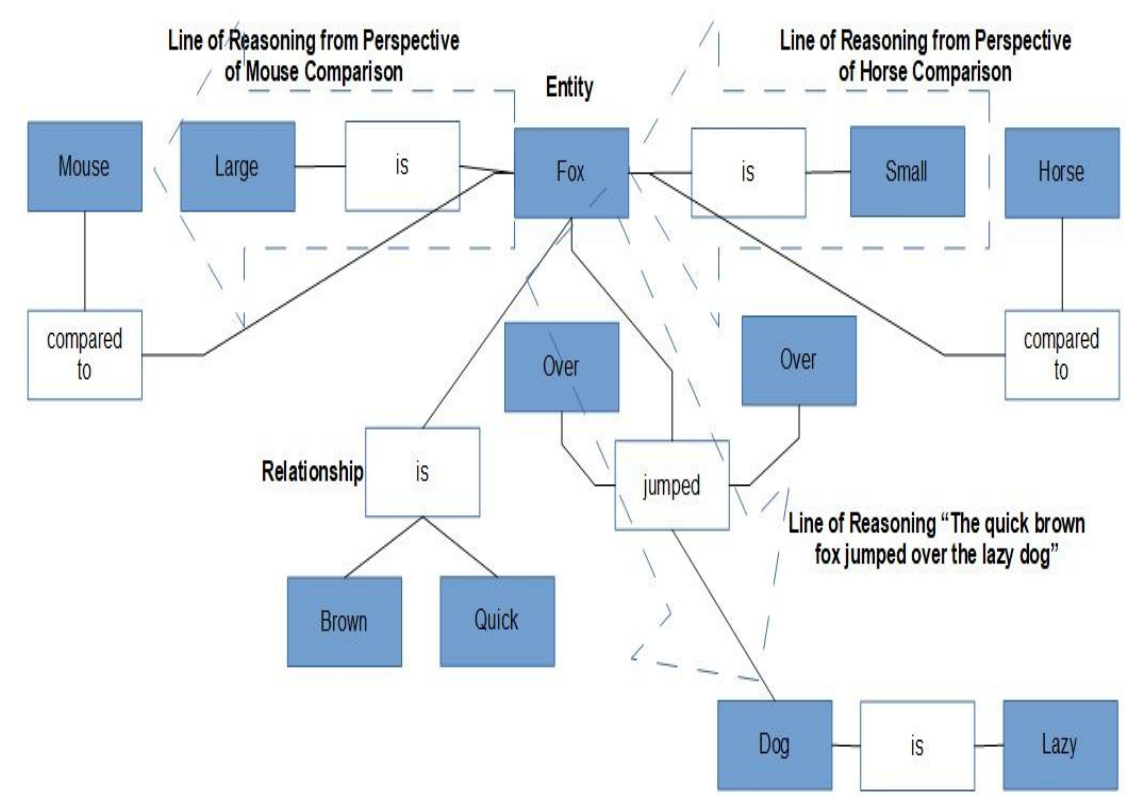

Figure 5: A zoom-in view of concepts in conceptual space.

The understanding process that produces the natural language sequence "the quick brown fox jumped over the lazy dog" can potentially be modeled in this case as a path through the region of conceptual space represented by the diagram above. Beginning at a position representing a given perspective on the entity "fox", and then executing the RECALL (F5) function on the properties "quick" and "brown" and executing the DETECT PATTERN (F6) function to associate them with the "fox", produces "the fox is quick" and "the fox is brown". The DETECT PATTERN (F6) might then associate "the fox is quick" with the "the fox is brown" through the pattern AND to produce "the fox is quick and brown". The process might then execute the DETECT SEQUENCE (F7) function to associate the sequence "the fox is quick and brown" with "the quick brown fox". The process might then execute the RECALL (F5) function on the relationship "jumped" to retrieve what the fox did. And then might execute the RECALL (F5) function on the modifier "over" to retrieve that information as well. Finally, it might execute the RECALL (F5) function on "lazy dog", and then execute the DETECT SEQUENCE (F7) function to group "the quick brown fox", "jumped over", and "the lazy dog". Reasoning or understanding processes, such as those required to construct text or speech in natural language, then become a sequence of paths through the conceptual space, where this sequence is represented in terms of the functional units. In this case, the first path P1 represented in the diagram below, is "the fox is quick". 


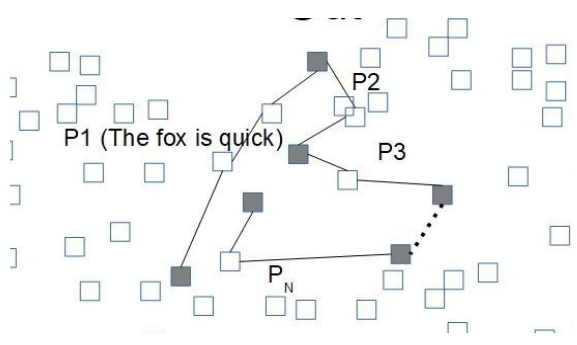

Figure 6: A zoom-out view of

concepts in conceptual space.

In order to be able to retrieve all the relationships relevant to a given perspective so that this path can be constructed, the relative positions of concepts in the conceptual space, as well as the relative positions of concepts defining these relationships, must together store information defining such perspectives. The representation of the conceptual space, including how to specify relationships between concepts, how to specify perspectives, and other elements necessary to implement this FMF, are ongoing subjects of research.

As mentioned, within this framework all functions in the cognitive system as well as all consciously observable functions in other parts of the human system, are modeled as adapting through adaptative processes $\mathrm{L} 1$ to $\mathrm{L} 8$, each of which is proposed to operate in a different adaptive domain. This decomposition into adaptive domains is intended to represent the ability of the human system to adapt each of its functions through these different processes, and its ability to reliably become more fit in each domain. In models of consciousness this adaptability is proposed to be required for models to have the capacity to become more fit so that they reliably converge on the functionality consciousness or cognition are observed to have.

As also mentioned, one domain of adaptability is cooperation. Within this framework the consciousness system, the cognitive system, as well as every other functional component having stably occuring functionality, are modeled as having to obey principles of intelligent cooperation P1 to P5 that are proposed to exist between the functional components implementing any functionality of the human system. These principles are intended to represent a balance between centralization and decentralization of processes, so processes can reliably achieve the capacity to maximize outcome for a group of functional components, and therefore operate in a stable way. The mechanisms of centralization are proposed to prioritize the function with the highest impact so it is possible to reliably maximize outcomes for the system. The mechanisms of decentralization are proposed to ensure outcomes are maximized for all functional components, rather than aligning outcomes with the interests of a subset of participants. Game theory based modeling [7] of these assumed forces suggests that without this balancing force of decentralization, decision-making naturally aligns with the interests of the subset of components acting as decision-makers, and that this alignment tends to constrain the group of components to solving the wrong problems so the systems can't reliably adapt to become more fit.

\section{Representing Models of Consciousness}

Where the cognitive system is proposed to navigate a virtual functional state space called here the conceptual space, the consciousness system is proposed to navigate an awareness space. Because the awareness space and conceptual space are abstract functional state spaces that are independent of implementation, because the conceptual space navigated by the cognitive system is open (unbounded), 
and because the awareness space navigated by the consciousness system is open as well, the set of functions required to navigate the awareness space is proposed in this framework to be the same, differing only in that they operate with awareness as inputs to and outputs from all processes and functions.

\begin{tabular}{|c|c|c|}
\hline \multicolumn{3}{|c|}{ Functional Units in Systems of Consciousness } \\
\hline Functional Unit & Input Function & Output Function \\
\hline $\mathrm{F} 1$ to $\mathrm{F} 3$ & Create Awareness & Create Signals from Awareness \\
\hline F4 & STORE (Store Awareness) & $\begin{array}{l}\text { DECOMPOSE STORAGE } \\
\text { (Determine Awareness in Storage } \\
\text { Function) }\end{array}$ \\
\hline F5 & RECALL (Recall Awareness) & $\begin{array}{l}\text { DECOMPOSE RECALL } \\
\text { (Determine Awareness in Recall } \\
\text { Function) }\end{array}$ \\
\hline F6 & $\begin{array}{l}\text { DETECT PATTERN (Detect } \\
\text { Pattern between Awarenesses) }\end{array}$ & $\begin{array}{l}\text { DECOMPOSE PATTERN (Detect } \\
\text { Awareness in Pattern) }\end{array}$ \\
\hline F7 & $\begin{array}{l}\text { DETECT SEQUENCE (Detect } \\
\text { Sequence of Patterns between } \\
\text { Awarenesses) }\end{array}$ & $\begin{array}{l}\text { DECOMPOSE SEQUENCE (Detect } \\
\text { Awareness in Sequence of Patterns) }\end{array}$ \\
\hline FS & $\begin{array}{l}\text { COGNITIVE AWARENESS (C } \\
\text { Awareness Process to Experienc }\end{array}$ & $\begin{array}{l}\text { loose Awareness to Experience and } \\
\text { e it) }\end{array}$ \\
\hline
\end{tabular}

Other Functional Components of the Human System in the Functional Modeling Framework The body receives inputs from the five senses. The body also processes sensory output into action. Receiving these five sets of inputs as the body acts is modeled in the FMF as the body moving through a functional state space defined in part by a sensory space. This sensory space is in turn composed of the tactile space, visual space, auditory space, gustatory space, and olfactory space. In addition, the body is represented as having some "fitness" (capacity to execute) each stable function it has. As described in the later sections of this paper, this fitness in the body is defined in the FMF as being related to physical well-being. Similarly, along with representing the mind as navigating a conceptual space, and the consciousness as navigating an awareness space as described earlier, the FMF represents the emotional system as navigating an emotional space. The representation of these state spaces is described elsewhere [61].

The capabilities of each functional system (again the body, emotions, mind, and consciousness) are represented as the set of processes and functions available within that specific system, each of which maps from one point in the system's perceptual space to another. In this modeling framework the metric of well-being of each functional system (its fitness) is its capacity to execute all its capabilities (functions). In the FMF this well-being is defined as being required to stay within some stable range. Models of each functional system can then identify whatever specific properties that in their model define the fitness space associated with that well-being, where the dynamics of the system are confined to that stable range in that fitness space, as indicated by the sphere below. 


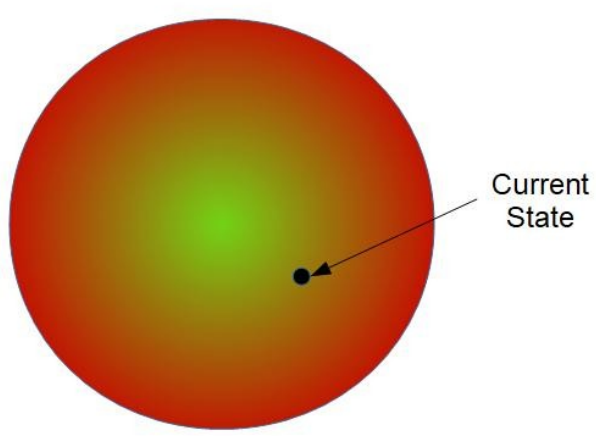

Figure 7: A graphical depiction of well-being remaining within a stable region.

\section{Integration of the Consciousness, Cognition, and Other Functional Systems in the Functional Modeling Framework}

Defining each functional system as having an awareness process FS through which it "chooses" which of its component processes to execute, each functional system is represented in the FMF as executing a sequence of those processes to navigate its perceptual space in a way that is sustainable with regards to the well-being of the system.

In the case of the mind, the mind is represented as selecting a sequence of reasoning processes with which to navigate the conceptual space. Each path through the conceptual space represents an outcome,

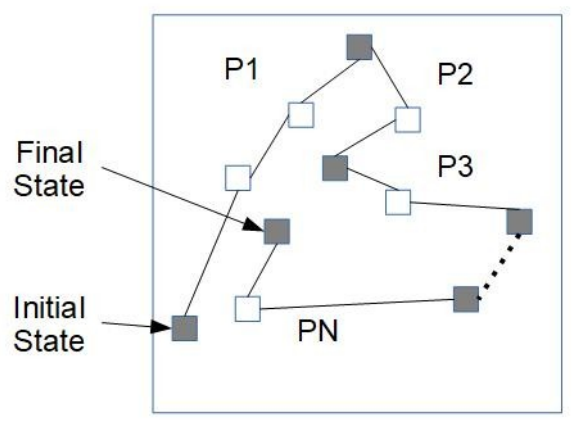

Figure 8: The sequence of processes navigates a conscious path through the perceptual field.

and the cost minus benefit of that outcome has an impact on mental well-being. This cost minus benefit and its impact on well-being are intended to be defined by models of cognition represented using this framework, rather than by the framework itself. Any path through the conceptual space must be nondeterministic since the cognitive system can respond to any random perturbation from the environment, that is, since the cognitive system (the mind), can conceptualize random events in the environment. At the same time the dynamics in fitness space must be globally stable throughout the entire conceptual space if the navigation is to continue.

These paths through the conceptual space are represented in the FMF as being integrated into a single awareness through the consciousness, which is represented as selecting a sequence of awareness 
processes with which to navigate the awareness space. In other words one awareness process might direct awareness to a reasoning process in the mind, another awareness process might direct awareness to a sensation in the body. If every physical, emotional, or cognitive perception can be represented as a point in a sensory, emotional, or conceptual state space, our ability to be aware of bodily, emotional, or mental perceptions indicates that human consciousness can have awareness processes that make it possible to be "aware" of potentially every point in every other perceptual space. In this sense, our consciousness process can be observed to switch it's focus from awareness of one system to another, like a spotlight moving to different areas on a stage.

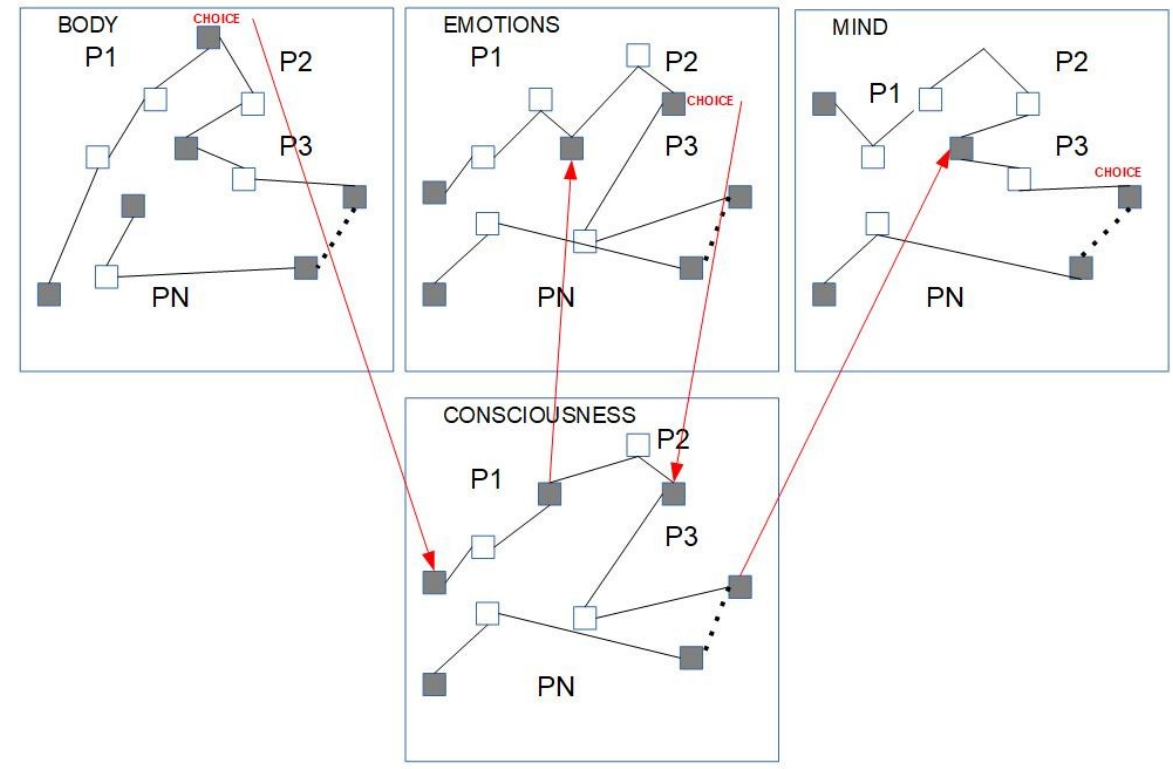

Figure 9: Conscious switching between processes.

Until an implementation replicates consciousness, this or any other framework for representing functional models of consciousness can't be confirmed to be complete. But a model of consciousness represented within this framework can be confirmed to be incomplete from the perspective of this framework. Because from this perspective, a model of consciousness that is not incomplete must have the capacity for this globally stable and therefore sustainable navigation of the awareness space. And to be self-aware, such a model must have the ability to direct its awareness to all other parts of the self (body, emotions, mind) in all of their states. Since the systems comprising the self, including their states, are represented in the FMF in terms of the perceptual spaces, and since the states of those systems can potentially be represented by any point in those perceptual spaces, then a model of consciousness that is not incomplete must also have the capacity to interact with potentially all points in the other perceptual spaces (sensory spaces, emotional space, and conceptual space) through its awareness processes. However, it's important to note that incompleteness of any given model does not equate to incorrectness or lack of usefulness. A partial model of consciousness might still be correct in simply representing part of the total required functionality. And even a partial model might add useful insight.

\section{Why Eight Awarenesses in the Functional Modeling Framework?}

Why is there a kind of awareness in each perceptual space (five types of sensory awareness and an awareness in the emotional, and conceptual space, as well as conscious self-awareness, for a total of what seem to be eight types of consciousness in this framework? 
The answer is that for each perceptual system, having the ability to navigate its perceptual space requires both perception of place in the perceptual space and choice of path from that place. That combination of perception and choice is represented here as awareness. Quick self-observation will confirm that each perceptual system can experience awarenesses outside the self-awareness of our consciousness (we can react unconsciously), so our awarenesses in each of these systems must be independent. Modeling each perceptual system as moving within the state space that we will call its perceptual space, the human system must navigate that perceptual space in a way that maintains a stable range of fitness. This paper proposes that there are two ways for biological processes to maintain a stable range of fitness in any functional state space, whether in the functional state space of a single cell, or in the collective functional state space of cells within an organ, or in the composite functional state space of the human system in which those functional components cooperate to maintain that fitness.

One way is to have a single stable path through the fitness space that might be highly complex, where this path through fitness space produces some path in functional state space. This scenario represents the complex path through state space that cells in a tree might take as they respond to their surrounding cells in ways causing them specialize into different parts of the tree. In this case a single complex path to homeostasis through fitness space might exist. In the range of states of the environment in which the organism can maintain stability, such non-animal organisms simply follow that path through their internal state space to "respond" to that environment.

The other way is to have a library of stable paths (a library of functions) through the fitness space that might be simpler, where each path through fitness space produces some path through functional state space, and to have a stable process of "choice" to select between them. The path through fitness space defined by the choice process, superimposed on the path through fitness space defined by each function chosen from the library, in this way has the potential to result in the emergence of ultra-complex behavior in the functional state space. This scenario represents the multiple actions that cells in an animal might choose in response to their environment. Or at the level of the organism, it represents the actions the organism might choose in response to a given perception. In this case, multiple paths to stability might exist. In the range of states of the environment in which the organism can maintain stability, the organism must "choose". To do so consciously it must perceive its position in the state space that defines the perceptual space. This combination of perceiving position in the perceptual space, and "choice" of action (choice of process representing a path in the space), is represented within the the FMF to be a kind of awareness that occurs in each perceptual space. In summary, awareness in a perceptual space is the capacity for navigation through that perceptual space in a way that is selfsustained in being globally stable through the associated fitness space.

Since the human-centric functional modeling of the FMF relies heavily on observations of the human system that can be made using only innate self-awareness, it is worthwhile noting that in terms of Eastern existential traditions of self-observation, the Yogācāra school of Mahayana Buddhism [6], reflects eight different consciousnesses which appear to be largely aligned with the above definitions.

\section{Differences from Other Modeling Approaches}

Many functions of the human system can be observed within individual self-awareness but can't yet be measured externally. Since this is true, any successful system of observation of the human system must rely at least in part on some methodology of internal measurement, that is, of observation through selfawareness. One of the functions of the human system is consciousness. The importance of developing models of understanding consciousness that can be validated with the individual's self-awareness is a long-established school of thought in the academic study of consciousness [5]. However this conflicts 
with what may be an even more prevalent school of thought, which is the idea that internal self observations are merely "anecdotal evidence", and therefore that excluding such observations and attempting to rely solely on external measurements of phenomena related to consciousness is a mark of "intellectual rigor".

The goal of unambiguously defining a complete set of the functions of consciousness is enabling models of consciousness to be represented and compared in an objective way. There have been a number of comparisons of models of consciousness outside this framework [45],[46],[47]. But the comparisons lack objective metrics with which they can be confirmed to converge on a common result. The kind of human-centric functional modeling framework proposed here may be required to be able to methodically compare all such models in common terms and get meaningfully similar conclusions from all comparisons as demonstrated through using this framework to compare some leading models of consciousness [8].

\section{The Usefulness of Functional Modeling in Creating Intuitive Models of Concepts such as Intelligence and Conscious Self-Awareness}

Beginning with the mind, considering concepts to be points in a "conceptual space", then a set of concepts describing anything is itself a single concept.

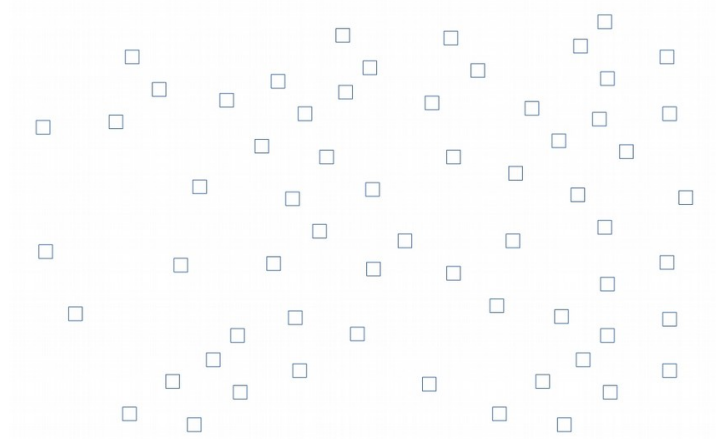

Figure 10: The conceptual space as one of other perceptual spaces (visual and other sensory spaces, as well as emotional and awareness spaces).

From this point of view any reasoning process requiring multiple concepts as inputs and producing multiple concepts as outputs is a path mapping one point in the conceptual space to another. A problem is then the lack of such a path. 


\section{Navigating The Perceptual Field}

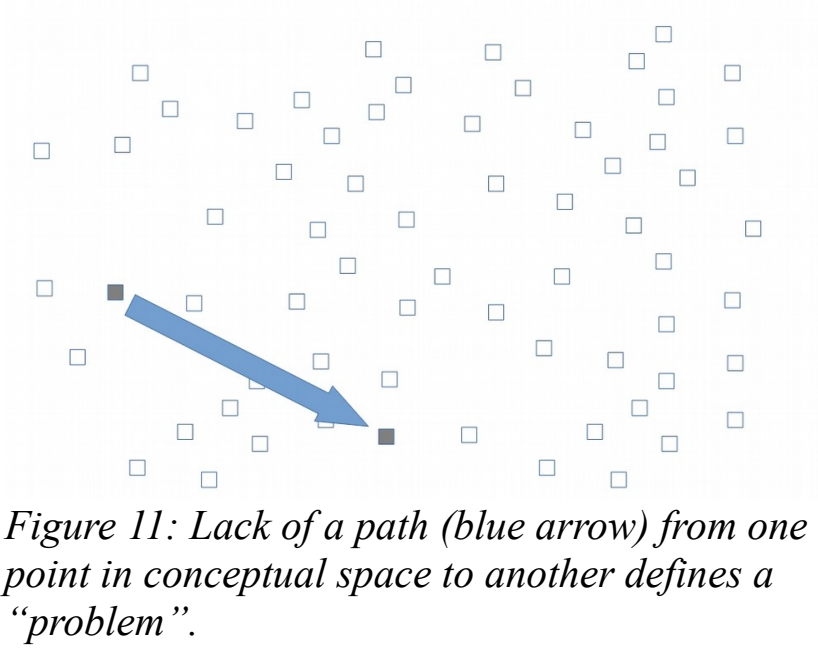

General problem solving ability from this perspective becomes a sequence of reasoning through which the mind can sustainably navigate the conceptual space in a way that potentially enables it to navigate from any concept to any other concept, that is, which gives the mind the ability to potentially solve any problem it can conceive.

The consciousness is similarly defined to navigate an awarenesses space. General self-awareness (conscious self-awareness) then becomes a sequence of awareness processes through which the consciousness can sustainably navigate the awareness space in a way that potentially enables it to navigate from any awareness to any other awareness in a self-sustaining way. That is, which gives the consciousness the ability to potentially solve the problem of navigating to any awareness it can perceive. In both the case of consciousness and of cognition, this sustainable navigation is proposed to reflect dynamical stability in some "fitness" space that in the case of consciousness is proposed to be related to conscious well-being. The mathematical relationship between human consciousness and dynamical stability has led others to also consider the relevance of concepts such as fractals and strange attractors in achieving that stability [17],[18],[19],[20].

This definition of perceptual spaces is "human-centric" in the sense that rather than requiring we assume validity of any theoretical framework, a human-centric definition requires nothing more than innate human awareness to validate. Researchers studying consciousness have defined a multitude of state spaces to describe consciousness and/or cognition [16]. However there are immense benefits in choosing this human-centric definition of consciousness and cognitive state spaces in terms of perceptual spaces that translate directly to the sensory inputs we perceive. One is that the FMF has been extremely successful in facilitating the mathematical expression of a wide range of attributes of human consciousness and cognition sometimes thought to be subjective and ambiguous, including level of intelligence (IQ). It has also been successful in defining a test for consciousness.

In the case of IQ, assume general problem solving ability is the ability to sustainably navigate the problem space so that it's potentially possible to navigate from any problem to its solution. And assume that remaining within a range of mental well-being is required to sustain that navigation so that there is a global stability in mental well-being across the entire conceptual space during this navigation. IQ is then proposed to be the volume of the problem space that can be navigated by the mind over the time frame of the typical test. However in order to be able to navigate to a region in conceptual space that 
region must be defined. Relative position of concepts in conceptual space is determined by conceptual relationships.
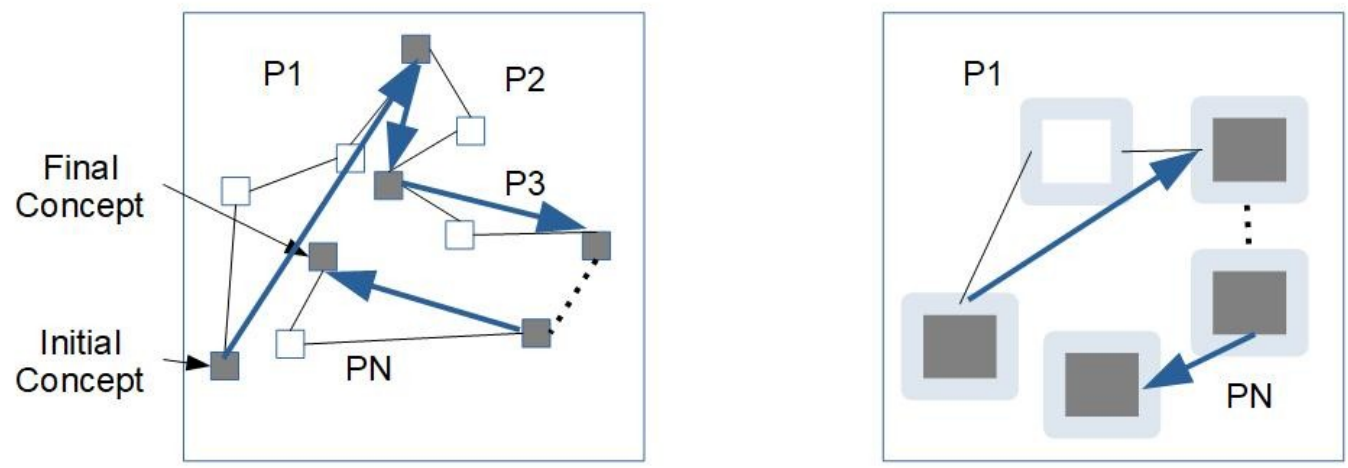

Figure 12: Concepts are defined by relationships that are in turn specified by reasoning paths through conceptual space. In the diagram to the left the cognitive system is able to detect the sufficient number of relationships required to resolve all the concepts in the reasoning process. In the FMF a problem is the gap between a starting concept and ending concept in the conceptual space. Solutions are the reasoning that provides the path between the two points. In the diagram to the right the cognitive system is unable to resolve concepts in sufficient detail to locate the start or end concepts, and therefore is unable to conceive of the problem or to conceive of the solution.

Grouping concepts into a reasoning process in this way enables one concept to be differentiated from another by function. The inability to group a sufficient number of concepts to resolve two positions in conceptual space as being separate limits reasoning processes to navigating from one uncertain concept covering a broader area in conceptual space, to another uncertain concept. Uncertainty is represented in the diagram on the right by the less sharply defined line border around the concept. With less ability to resolve concepts, the effective area in the conceptual space that can be navigated, and therefore the general problem solving ability of the cognitive system (it's intelligence), are greatly reduced.

In this model a similar global stability is defined for the path of the consciousness system through the awareness space. Just as a test for intelligence in this model is proposed to be the ability of the mind to sustainably navigate it's conceptual space, and IQ is the volume of conceptual space navigable in the time of an IQ test, a test for consciousness in this model is proposed to be the ability to sustainably navigate the awareness space. And like IQ, the level of consciousness, or from the point of view of Eastern existential philosophies like the yogic system, what they might call the degree of enlightenment, then becomes the volume of the awareness space that can be navigated per unit time in order to arrive at an awareness that solves the problem of conscious well-being.

\section{Properties of Valid and Complete Models of Consciousness or Cognition in the FMF}

For models of cognition to be complete and valid within this framework they must display this global stability in fitness space despite local instability in conceptual space. And they must display the ability to adapt to become more fit in cognition. And for models of consciousness to be complete and valid within this framework they must also display this global stability despite local instability, as well as this adaptability, that is required of all processes in this framework. 


\section{Conclusions}

This paper states and attempts to provide some justification for the key elements of a framework for defining and comparing functional models of consciousness or cognition. The list of functional units, adaptive processes (basic life processes), principles of cooperation, and other functional components stands to benefit from further validation. However, the most important takeaway from the point of view of consciousness or cognition studies is that basic elements of the framework might be used now to provide the ability to compare models of consciousness, and to provide the ability to take the most fit components of each model so that overall understanding converges on the actual functionality observed in consciousness and cognition.

These modeling elements have already been used to compare some leading models of consciousness [8]. Because having a common framework for comparison can potentially enable each model of consciousness to reuse the best components of each other model, implementations of the components of the FMF by such models can then potentially form valuable research infrastructure with the potential to radically increase the impact and accelerate the progress of any group working on defining such models. The plans being developed for a prospective series of paralel LSCORE (Large Scale international COllaborative Research) initiatives proposes to leverage the FMF to organize cooperation between a wide range of projects so that they form this infrastructure. Through creating the possibility of such massive collaboration this initiative aims to become the CERN of consciousness and cognition research.

Furthermore, in defining all living functions as potentially sharing a global stability in their fitness space, despite a potentially chaotic and unstable path through their functional state space, this framework may identify a single pattern that enables all biological functions, and life itself, to potentially be viewed as variations of the same solution that life has developed for achieving stability in different contexts over the 3.5 billion year history of the earth. This potentially enables models of consciousness to be viewed as solutions that can be reused in a much wider variety of problems. And potentially, as explored elsewhere [8], as a mathematical pattern of stability that has its origins in the laws of physics themselves.

\section{References}

[1] Model for Human, Artificial \& Collective Consciousness (Part I), Journal of Consciousness Exploration \& Research, Andy E. Williams, Vol 10, No 4 (2019), pages 250-269

[2] Model for Human, Andy E. Williams, Artificial \& Collective Consciousness (Part II), Journal of Consciousness Exploration \& Research, Vol 10, No 4 (2019), pages 270-293

[3] The Relationship Between Collective Intelligence and One Model of General Collective Intelligence, Andy E. Williams, Computational Collective Intelligence, 11th International Conference, ICCCI 2019, Hendaye, France, September 4-6, 2019, Proceedings, Part II, Pages 589-600

[4] Andy E. Williams, A Model for General Collective Intelligence, under review, 2019

[5] Varela, F. J., Thompson, E., and Rosch, E. The Embodied Mind: Cognitive Science and Human

Experience. Cambridge, MA: MIT Press, 1991.

[6] Harivarman. "Sātyasiddhiśāstra"At vargaḥ 62 (nācaitasikavargaḥ): "ya ādhyātmiko 'sti vijñānakāyāḥ"

[7] Andy E. Williams, The Principles Enabling Cooperation within Conscious Organisms and Collectives, working paper.

[8] Andy E. Williams, Using a Functional Modeling Framework to Compare Some Leading Models of Consciousness, working paper. 
[9] Tononi, Giulio; Boly, Melanie; Massimini, Marcello; Koch, Christof (2016). "Integrated information theory: from consciousness to its physical substrate". Nature Reviews Neuroscience. 17 (7): 450-461. doi:10.1038/nrn.2016.44. PMID 27225071.

[10] Penrose, Roger (2014). "On the Gravitization of Quantum Mechanics 1: Quantum State Reduction". Foundations of Physics. 44 (5): 557-575. Bibcode:2014FoPh...44..557P. doi:10.1007/s10701-013-9770-0.

[11] Andy E. Williams, The eXtensible Domain Modeling Framework (XDMF), working paper.

[12] Andy E. Williams, A Bridge Between Computer Science and Social Innovation Approaches to Collective Intelligence, working paper.

[13] Andy E. Williams, The Universal Impact Metrics Framework (UIMF), working paper.

[14] Andy E. Williams, The Semantic Metrics Framework (SMF), working paper.

[15] Andy E. Williams, A Model for Artificial General Intelligence (AGI), under review.

[16] Berkovich-Ohana, Aviva, and Joseph Glicksohn. "The consciousness state space (CSS)-a unifying model for consciousness and self." Frontiers in psychology vol. 5341.29 Apr. 2014, doi:10.3389/fpsyg.2014.00341

[17] Bielecki A1, Kokoszka A, Holas P., Dynamic systems theory approach to consciousness., Int J Neurosci. 2000 Sep-Oct;104(1-4):29-47.

[18] Chris King, Fractal Neurodyamics and Quantum Chaos : Resolving the Mind-Brain Paradox Through Novel Biophysics, in In Fractals of Brain Fractals of Mind, Advances in Consciousness Research 7, John Betjamin \& Co. 1996

[19] Perlovsky L.I., Kozma R. (2007) Neurodynamics of Cognition and Consciousness. In: Perlovsky L.I., Kozma R. (eds) Neurodynamics of Cognition and Consciousness. Understanding Complex Systems. Springer, Berlin, Heidelberg [20] Larry R. Vandervert, Chaos theory and the evolution of consciousness and mind: A thermodynamic-holographic resolution to the mind-body problem, New Ideas in Psychology, Volume 13, Issue 2, 1995, Pages 107-127, ISSN 0732-118X, https://doi.org/10.1016/0732-118X(94)00047-7. [21] B. CHANDRASEKARAN, Representing function: Relating functional representation and functional modeling research streams, Artificial Intelligence for Engineering Design, Analysis and Manufacturing 2005, 19, 65-74, DOI: 10.10170S0890060405050079

[22] Zhang, W.Y., Tor, S.Y. \& Britton, G.A. , Managing modularity in product family design with functional modeling, The International Journal of Advanced Manufacturing Technology(2006) 30: 579. https://doi.org/10.1007/s00170-005-0112-z

[23] Eisenbart, B., Gericke, K., \& Blessing, L. (2013). An analysis of functional modeling approaches across disciplines. Artificial Intelligence for Engineering Design, Analysis and Manufacturing, 27(3), 281-289. doi:10.1017/S0890060413000280

[24] Zhengrong Cheng, Yongsheng Ma, A functional feature modeling method, Advanced Engineering Informatics, Volume 33, 2017, Pages 1-15, ISSN 1474-0346, https://doi.org/10.1016/j.aei.2017.04.003. [25] Tensa, M., Edmonds, K., Ferrero, V., Mikes, A., Soria Zurita, N., Stone, R., \& DuPont, B. (2019). Toward Automated Functional Modeling: An Association Rules Approach for Mining the Relationship between Product Components and Function. Proceedings of the Design Society: International Conference on Engineering Design, 1(1), 1713-1722. doi:10.1017/dsi.2019.177

[26] Sten Bay Jørgensen, Morten Lind, and Niels Jensen, Functional Modeling View on Product and Process Engineering in Design and Operations

Industrial \& Engineering Chemistry Research 201958 (26), 11129-11148

DOI: $10.1021 /$ acs.iecr.8b06338

[27] Zhao, Meng, et al. "A State-Behavior-Function Model for Functional Modeling of Multi-State Systems." Proceedings of the Institution of Mechanical Engineers, Part C: Journal of Mechanical Engineering Science, vol. 233, no. 7, Apr. 2019, pp. 2302-2317, doi:10.1177/0954406218791640. 
[28] Christoffer Levandowski, Jakob R Müller, Ola Isaksson, Modularization in Concept Development Using Functional Modeling, Transdisciplinary Engineering: Crossing Boundaries: Proceedings of the 23rd ISPE Inc. International Conference on Transdisciplinary Engineering October 3 - 7, 2016 [29] P Bruyndonckx, S Léonard, S Tavernier, Neural network-based position estimators for PET detectors using monolithic LSO blocks, IEEE Transactions on Nuclear Science, 2004 ieeexplore.ieee.org [30] IE Ebong, P Mazumder, CMOS and Memristor-Based Neural Network Design for Position Detection. Proceedings of the IEEE, 2012 - ieeexplore.ieee.org

[31] I Sutskever, O Vinyals, Sequence to sequence learning with neural networks, QV Le - Advances in Neural Information Processing Systems 27 (NIPS 2014) - papers.nips.cc

[32] G Houghton, The problem of serial order: A neural network model of sequence learning and recall - Current research in natural language generation, 1990 - dl.acm.org

[33] S Nara, P Davis, H Totsuji, Memory search using complex dynamics in a recurrent neural network model, Neural Networks, 1993 - Elsevier

[34] MA Cohen, S Grossberg, Absolute stability of global pattern formation and parallel memory storage by competitive neural networks - IEEE Transactions on Systems, Man, and Cybernetics, 1983 ieeexplore.ieee.org

[35] K Yao, B Peng, Y Zhang, D Yu, Spoken language understanding using long short-term memory neural networks, 2014 IEEE Spoken Language Technology Workshop (SLT) -ieeexplore.ieee.org [36] IS Aranson, A Pikovsky, NF Rulkov, Advances in Dynamics, Patterns, Cognition, LS Tsimring 2017 - Springer

[37] J Sietsma, RJF Dow, Creating artificial neural networks that generalize, Neural networks, 1991 Elsevier

[38] Pattaranit, Ratchada, and Hugo Antonius van den Berg. "Mathematical models of energy homeostasis." Journal of the Royal Society, Interface vol. 5,27 (2008): 1119-35.

doi:10.1098/rsif.2008.0216

[39] Jiangjun Cui, Jaap A. Kaandorp, Mathematical modeling of calcium homeostasis in yeast cells, Cell Calcium, Volume 39, Issue 4, 2006, Pages 337-348, ISSN 0143-4160, https://doi.org/10.1016/j.ceca.2005.12.001.

[40] Weinstein, A.M. , Dynamics of cellular homeostasis: Recovery time for a perturbation from equilibrium, Bulletin of Mathematical Biology (1997) 59: 451. https://doi.org/10.1007/BF02459460 [41] Hannah Schramke, Filipe Tostevin, Ralf Heermann, Ulrich Gerland, Kirsten Jung, A Dual-Sensing Receptor Confers Robust Cellular Homeostasis, Cell Reports, Volume 16, Issue 1, 2016, Pages 213 221, ISSN 2211-1247, https://doi.org/10.1016/j.celrep.2016.05.081.

[42] Yasuaki Kobayashi, Yusuke Sawabu, Hiroyuki Kitahata, Mitsuhiro Denda, Masaharu Nagayama, Mathematical model for calcium-assisted epidermal homeostasis, Journal of Theoretical Biology, Volume 397, 2016, Pages 52-60, ISSN 0022-5193, https://doi.org/10.1016/j.jtbi.2016.02.032. [43] Lu Xia, Jin Guantao, Chen Wenjin, Yu Xinguang, Ling Feng, Depiction of Physiological Homeostasis by Self-Coupled System and Its Significance, Frontiers in Physiology, vol 10, 2019, page 1205, DOI=10.3389/fphys.2019.01205

[44] A. L. Ramos, J. V. Ferreira and J. Barceló, "LITHE: An Agile Methodology for Human-Centric Model-Based Systems Engineering," in IEEE Transactions on Systems, Man, and Cybernetics:

Systems, vol. 43, no. 3, pp. 504-521, May 2013.

doi: 10.1109/TSMCA.2012.2207888

[45] Block, N. (2009). Comparing the major theories of consciousness. In M. S. Gazzaniga, E. Bizzi, L. M. Chalupa, S. T. Grafton, T. F. Heatherton, C. Koch, ... B. A. Wandell (Eds.), The cognitive neurosciences (pp. 1111-1122). Cambridge, MA, US: Massachusetts Institute of Technology. [46] Snezana Cerepnalkovska Dukovska, Comparison on models of consciousness: Hameroff-Penrose/ Henry Stapp/ Berner-Percinkova, Proceedings of ICT-ACT Innovations 2013, Ohrid 
[47] Ravinder Jerath, Connor Beveridge, Critique and Comparison of Prevailing Consciousness Models with a Novel Embodied Cognition Model, WJNS Vol.8 No.3, August 2018 [48] Bronfman, Z.Z.; Ginsburg, S., The Evolutionary Origins of Consciousness: Suggesting a Transition Marker, Journal of Consciousness Studies, Volume 23, Numbers 9-10, 2016, pp. 7-34(28) [49] Max Velmans, How could consciousness emerge from adaptive functioning?, Animal Sentience 2016.138: Velmans on Reber on Origins of Mind [50] Birch, J. Acta, In Search of the Origins of Consciousness, Biotheor (2019). https://doi.org/10.1007/s10441-019-09363-x [51] David Rudrauf, Daniel Bennequin, Isabela Granic, Gregory Landini, Karl Friston, Kenneth Williford, A mathematical model of embodied consciousness, Journal of Theoretical Biology, Volume 428, 2017, Pages 106-131, ISSN 0022-5193, https://doi.org/10.1016/j.jtbi.2017.05.032.

[52] De Bruijn, N. G., A Mathematical Model for Biological Memory and Consciousness, Thirty Five Years of Automating Mathematics, 2003, Springer Netherlands, Dordrecht [53] Hamidreza Namazi, Vladimir Vladimirovich Kulish, A mathematical based definition of human consciousness, Mathematics in Engineering, Science and Aerospace, Vol 3 No 2 (2012)

[54] Willard L. Miranker, Gregg J. Zuckerman, Mathematical foundations of consciousness, Journal of Applied Logic, Volume 7, Issue 4, 2009, Pages 421-440, ISSN 1570-8683, https://doi.org/10.1016/j.jal.2008.05.002.

[55] Federica Stefanelli, Enrico Imbimbo, Timoteo Carletti, Alessio Guarino, Francois Burette, et al.. A mathematical model of Collective Intelligence. 2019. ffhal-02059353v2

[56] Andy E. Williams, The Clinical Benefits of Human-Centric Modeling of Consciousness and Cognition, working paper.

[57 R Jerath, C Beveridge, Top Mysteries of the Mind: Insights From the Default Space Model of Consciousness

Frontiers in human neuroscience, 2018 - frontiersin.org

[58] Ravinder Jerath, Molly W. Crawford, and Vernon A. Barnes

A unified 3D default space consciousness model combining neurological and physiological processes that underlie conscious experience

Front Psychol. 2015 Aug 27;6:1204. doi: 10.3389/fpsyg.2015.01204. ECollection 2015.

[59] Andy E. Williams, Using Information Theory and Non-Equilibrium Dynamics in the Flow of Entropy to Derive the Functional Components of Consciousness and Cognition, working paper [60] Kahneman, D. (2012).Thinking, fast and slow.London: Penguin Books. Isbn: 0141033576. [61] Andy E. Williams, A Functional Model for Sensory Perception as the Origin of Conscious Awareness and Cognitive Conceptualization, working paper [62] Andy E. Williams, Functional Decomposition, working paper 